RESEARCH NOTE

\section{Immune Response During HIV and Tuberculosis Co-infection}

\section{MG Bonecini-Almeida/ ${ }^{+}$, JR Lapa e Silva*, AL Kritski**, I Neves Jr., MG Morgado, C Nathan***, JL Ho****}

Laboratório de AIDS e Imunologia Molecular, Departamento de Imunologia, Instituto Oswaldo Cruz, Av. Brasil 4365, 21045-900 Rio de Janeiro, RJ, Brasil *Laboratório Multidisciplinar de Pesquisa **Serviço de Pneumologia, Hospital Universitário Clementino Fraga Filho, Universidade Federal do Rio de Janeiro, RJ, Brasil $* * *$ Beatrice and Samuel A Seaver Laboratory, Division of Hematology-Oncology ****Division of International Medicine and Infectious Diseases, Department of Medicine, Cornell University Medical College, New York, NY, USA

Key words: tuberculosis - HIV - macrophage cytokines and cell activation

The human immunodeficiency virus type 1 (HIV-1) and Mycobacterium tuberculosis, the etiologic agent of tuberculosis (TB), co-infect around 6 million people worldwide (WHO 1992, Report 24-26 February). In Rio de Janeiro, Brazil, 24\% of notified AIDS cases had TB and 5 to $20 \%$ of notified TB cases are HIV-1 seropositive. Several authors have already described the deleterious association between these two microorganisms (SB Mannoff et al. 1996 J Infect Dis 174: 299-308, F Pulido et al. 1997 Arch Intern Med 157: 227-232, V Leroy et al. 1997 Am J Epid 145: 293-300, PL Alpert et al. 1997 Clin Infect Dis 24: 661-668). Here, we will overview the immune response to M. tuberculosis and the effect of association with HIV-1 infection. The natural history of M. tuber-

Supported by grants from UNDP/World Bank/Brazilian Ministry of Health-STD/AIDS Program (21/94, 03/95 and 12/96), Brazilian Council of Research - CNPq and by National Institutes of Health (AI34543, R37-22624 and D43-TW000181).

${ }^{+}$Corresponding author. Fax: +55-21-280.1589. E-mail: galmeida@gene.dbbm.fiocruz.br

Received 11 March 1998

Accepted 16 April 1998 culosis infection indicates that the emergence of delayed-type hypersensitivity (DTH) and presumably specific acquired resistance is associated with control of the initial infection in $95 \%$ of normal hosts; the other $5 \%$ develop progressive primary TB. In addition, $5-10 \%$ of the infected persons eventually will reactivate latent pulmonary or extrapulmonary foci several years after infection (MC Raviglione et al. 1995 JAMA 272: 220-226). HIV-infected individuals and AIDS patients have a remarkable susceptibility to TB, increasing 113fold and 170-fold the risk of TB reactivation, respectively (ME Villarino et al. 1992 Morb Mortal Wkly Rep 41: 61-65). In addition, it has been shown that TB accelerates the HIV infection and disease progression (JW Pape et al. 1993 Lancet 342: 268271, C Whalen et al. 1995 Am J Respir Crit Care Med 151: 129-133).

Cells belonging to the monocyte/macrophage lineage are important target cells for HIV and $M$. tuberculosis, and there is increasing evidence that these cells play a crucial role in the pathogenesis of these intracellular infections. Their susceptibility to HIV infection in vivo has been demonstrated in brain (A Porwit et al. 1989 APMIS 97: 79-90), spinal cord (TM Folks et al. 1988 Science 242: 919922), lymphonode (F Plata et al. 1987 Nature 328: 348-351) and lung (GU Meduri et al. 1992 Clin Infect Dis 14: 98-113). HIV-1 also readily infects human monocyte/macrophage in vitro (MS Meltzer et al. 1990 Am Rev Immunol 8: 169-194, HS Nottet et al. 1993 J Infect Dis 167: 810-817). Being target to both mycobacteria and virus, the monocyte/ macrophage lineages change their functional activities after double infection. Concurrent infection of human macrophages with HIV-1 and $M$. avium results in decreased cell viability, increased bacilli multiplication and altered cytokine production in vitro (GW Newman et al. 1993 J Immunol 151: 2261-2272). Z Toossi et al. (1993 J Exp Med 177: 1511-1516) demonstrated that peripheral blood monocytes from patients with active pulmonary tuberculosis are more susceptible to productive infection with HIV-1, leading to an increase in peripheral (D Golett et al. 1996 J Immunol 157: 12711278) and lung (K Nakata et al. 1997 Am J Respir Crit Care Med 155: 996-1003) virus replication. Interestingly, the primordial function of monocyte/ macrophage, the phagocytosis of $M$. tuberculosis, enhances the transcription of HIV LTR (RJ Shattock et al. 1993 Res Virol 144: 7-12) in vitro. Since HIV1 and M. tuberculosis share the same target cells in a specific microenvironment, the lungs, we have studied the interaction of these two pathogens in human co-infected individuals. Results from our group showed a reduction of $50-$ and $52.5 \%$ in phagocytic activity of alveolar macrophages from 
AIDS $(33 \pm 3.5 \%$ versus $66 \pm 7.8 \%)$ and AIDSTB $(39.9 \pm 13.1 \%$ versus $76 \pm 9.9 \%)$ patients, respectively, when compared to peripheral monocytes $(\mathrm{p}<0.05)$. TB and healthy control individuals did not show any alteration in the monocyte/ macrophage phagocytic ability (MG BoneciniAlmeida et al. 1998, unpublished results).

Moreover, persons newly infected with $M$. tuberculosis and who have HIV co-infection have developed active TB disease more rapidly than HIV-uninfected persons. Otherwise, the HIV-associated accelerating of TB was alarmingly demonstrated in institutional outbreaks of multidrugresistant TB (CDC 1990 Morb Mortal Wkly Rep 39: 718-722, CDC 1991 40: 585-591).

These studies have added greatly to our understanding of the role of M. tuberculosis, particularly in monocyte/macrophage lineages, enhancing cytokines release that stimulate HIV replication (J Kekow et al. 1990 Proc Natl Acad Sci USA. 87: 8321-8325, A Fauci 1996 Nature 384: 529-531); however, immunological resistance and susceptibility to facultative intracellular bacterial pathogens depends also on T lymphocytes, natural killer cells and the macrophage regulatory cytokines released by these cells.

Tuberculosis disease causes in immunocompetent subjects a decreased peripheral lymphoproliferative response against $M$. tuberculosis-antigens and induces proliferation of blood and alveolar $\mathrm{T}$ lymphocytes from healthy PPD-positive subjects. As expected for a population in which bacillus Calmette-Guerin (BCG) vaccination is mandatory, such as the Brazilian population, blood and alveolar $\mathrm{T}$ lymphocytes from a healthy control group responded to PPD (PBMC, $8.3 \pm 0.9$; BAL, $9.8 \pm$ 1.2) and H37Ra-M. tuberculosis antigens (PBMC, $7.3 \pm 1.3$; BAL, $10.9 \pm 1.5$ ) in a similar manner. Also, a reduction in the lymphoproliferative response of blood and alveolar T lymphocytes against PPD-antigen in AIDS (PBMC, $1.5 \pm 0.3$; BAL, $2.1 \pm 0.5$ ) and AIDS/TB patients (PBMC, $3.1 \pm$ 0.8 ; BAL, $2.6 \pm 0.8$ ) was observed; however, no significant statistical difference was observed between these microenvironments. Recent study have demonstrated that HIV-positive patients with tuberculosis have a reduced enrichment and activation of immune cells in the lung, and the failure of $\mathrm{CD} 4^{+} \mathrm{T}$ lymphocytes alveolitis limits an effective immune response (KF Law et al. 1996 Am J Respir Crit Care Med 153: 1377-1384). Analyzing the alveolar cell profile on TB HIV-positive or -negative patients we noted an inversion of the $\mathrm{CD}^{+}$/ $\mathrm{CD}^{+}$ratio in all AIDS patients, with $($ratio $=0.50)$ or without (ratio $=0.21$ ) infectious pneumopathy or TB (ratio $=0.29$ ), reflecting in the lungs the peripheral depletion of $\mathrm{CD}^{+} \mathrm{T}$ cells. TB patients showed a 2.5 -fold decrease in $\mathrm{CD}^{+}{ }^{+} \mathrm{T}$ cells $(14.6$ $\pm 8.6 \%$ ) compared with the healthy control group $(36.8 \pm 2.6 \%, p<0.05)$ (Bonecini-Almeida et al. 1998, unpublished results).

In patients infected with M. leprae, $\mathrm{CD}^{+} \mathrm{T}$ type 1 (Th1) cells that produce IFN-g and IL-2 predominate in tuberculoid leprosy patients, whereas Th2 cells producing IL-4 and IL-10 are predominant in lepromatous leprosy patients with ineffective immunity (M Yamamura et al. 1991 Science 254: 277-279). The relative importance of cytokine production by human $\mathrm{CD} 4^{+} \mathrm{T}$ lymphocytes is still unclear in TB. Some authors described that most $\mathrm{CD}^{+} M$. tuberculosis-reactive $\mathrm{T}$ cells propagated in vitro are Th1 like with low production of IL-4 and IL-10 (GF Del Prete et al. 1991 J Clin Immunol 88: 346-350, JBAG Haanen et al. 1991 J Exp Med 174: 583-592) while others noted a mixed or less restricted TH0-like pattern (i.e., IFN-g, IL-10, or TNF-a in combination with IL-2, IL-5 or both) (WH Boom et al. 1991 Infect Immun 59: 2737-2743, PF Barnes et al. 1993 Infect Immun 61: 197-203; P Mendezsamperio et al. 1995 Cell Immunol 162: 194-201). Because of this discrepancy it is very important to analyze cytokine expression at the site of infection. In patients with tuberculous pleuritis the Th1 cytokine mRNA expression is higher than in blood; however, the Th2 cytokine expression was lower in pleural fluid than blood (PF Barnes et al. 1993 Infect Immun 62: 5673-5678). Thus, the role of an enhanced Th2 response in tuberculosis patients remains uncertain. The progression to AIDS leads to a progressive $\mathrm{CD} 4^{+}$depletion increasing the risk for TB disease (BE Jones et al. 1993 Am Respir Dis 148: 1292-1297). Activation of $\mathrm{CD}^{+}$by exposure with $M$. tuberculosis results in production of cytokines, many of which enhance HIV replication. Dual human infected macrophages in response to PPD (RS Wallis et al. 1993 J Infect Dis 167: 43-48) and double infected patients (N Boechat et al. 1997 Am J Respir Crit Care Med 155: A338) produce more TNF than TB or HIV alone. This cytokine is shown to promote HIV replication (T Matsuyama et al. 1991 AIDS 5: 1405-1417). The Th1/Th2 regulatory control of the immune system is still controversy in HIV-1 infection (R Manetti et al. 1996 Chem Immunol 63: 138-157), whereas the dual infection may also modify the cytokine expression. Recently, a study described the stimulation of PBMC from TB patients, with or without HIV infection, with M. tuberculosis in vitro, and evaluated the production and expression of Th1 and $\mathrm{Th} 2$ cytokines. They described a reduction of Th1 cytokines in PBMC from patients co-infected with HIV, whereas mRNA for Th2 cytokines was not 
reduced (M Zhang et al. 1994 J Clin Invest 94: 2435-2442). These results suggest that HIV decreases Th1 cytokines production, but does not enhance Th2 responses.

An alternative approach to evaluate the real relationship of the regulatory cytokine response in active pulmonary TB and the HIV implication is to compare the cytokine expression on alveolar cells. We found, in a preliminary study, that the expression of at least one of the activating Th1 cytokines (IL-2 and IFN-g) was seen in 19 of the 21 bronchoalveolar lavage (BAL) lysates from tuberculosis patients, independent of HIV serologic status. Simultaneous expression of the potentially deactivating Th2 cytokines (IL-4 and IL-10) was detected in 17 of the 19 lysates tested, where $84.2 \%$ (16 of 19) of the samples from TB patients expressed IL-10 mRNA. Presence of mRNA for IL-4 was consistently greater in $53.3 \%$ (8 of 15 ) of BAL cell lysates from tuberculosis patients, regardless of HIV status, whereas IL-4 expression was present in $28.6 \%$ of BAL samples from AIDS nonTB patients and in $16.6 \%$ of BAL samples from patients with other lung disease than TB and HIV infection (JR Lapa-Silva et al. 1997 Am J Respir Crit Care Med 155: A337).

Understanding the balance between Th1/Th2 cytokine like IFN-g, the most important and intensively studied cytokine that can activate antimycobacterial mechanisms of murine mononuclear phagocytes (S Huang et al. 1993 Science 259: 1742-1745, IM Orme et al. 1993 J Immunol 151 :518-524, SH Kaufmann et al. 1996 Ciba Found Symp 195: 123-132) and human macrophages in vitro (MG Bonecini-Almeida $1998 \mathrm{~J}$ Immunol in press); and IL-4, a macrophage deactivating cytokine (HM Naif et al. $1997 \mathrm{~J}$ Immunol 158: 501-511), may lead us to control or inhibit M. tuberculosis growth and HIV replication, or even improve the double infected patients clinical outcome.

Recently, we demonstrated that IFN-gis present in alveolar space from TB patients, and this cytokine alone, or together with lipopolysaccharide or TNF-a can activate murine macrophages to kill or inhibit mycobacteria by the induction of inducible nitric oxide synthase (iNOS) (J Chan et al. 1992 J Exp Med 175: 1111-1122, JD MacMicking et al. 1997 Proc Natl Acad Sci 94: 52435248). Nitric oxide (NO) has been directly related to the capacity of IFN-gactivated murine macrophages to kill M. tuberculosis, M. avium or M. leprae (IEA Flesh et al. 1990 Infect Immun 58: 2675-2677, M Denis et al. 1991 J Leuk Biol 49: 380-387, Chan et al. loc. cit., MacMicking loc. cit.). Moreover, inhibition of NO production in vivo increased mortality, bacillary burden, and tissue damage in mice infected with virulent $M$. tuberculosis (Chan et al. loc. cit.). The role of IFN-g and NO in protective immunity to mycobacterial infections in humans is poorly defined. S Nicholson et al. (1996 J Exp Med 183: 2293-2302) studying alveolar macrophages showed an average of $65 \%$ of these cells from all TB patients being positive for iNOS (NOS2). In contrast, only a mean of $10 \%$ of BAL cells were positive in healthy subjects. The in vivo co-expression of iNOS and cytokines during active TB is not well delineated. We demonstrated (MG Bonecini-Almeida et al. 1997 Am J Respir Crit Care Med 155: A441) that in alveolar cells from 9 of $11(81 \%)$ TB patients expression of iNOS, IFN$g$ and Th2-like cytokines were observed, suggesting that the levels of iNOS activity or other host or microbial factors may be involved in restriction of M. tuberculosis infection and dissemination, disease formation and reactivation.

HIV-infection induces iNOS expression on human microglia and neurons infected in vitro (DC Adamson 1996 Science 274:1917-1921) and NOproduced by iNOS is thought to induce dementia in HIV-positive individuals (Adamson et al. loc. cit.). Analyzing BAL cells from AIDS and AIDS/ TB patients we observed, in a preliminary study, that 5 of $6(83.3 \%)$ and 3 of $4(75 \%)$ patients expressed iNOS. Interestingly, $50 \%$ of AIDS/TB patients co-expressed IL-4.

Ideally, DTH to PPD could be used as a surrogate marker of protection, in non endemic countries and where BCG vaccination was not applied to control TB. However, in areas of high transmission like Rio de Janeiro with an incidence of 160 per 100,000 habitants, this issue should be reviewed. Analysis of BCG trials indicates that DTH is not predictive of protection (GW Comstock et al. 1988 Am Rev Respir Dis 138: 79-80). Preliminarily, we have been analyzing in individuals with high risk transmission (household contacts - HC), regarding the HIV serum status, $\mathrm{CD} 4^{+} \mathrm{T}$ cell count, previous BCG vaccination, PPD skin test and $\mathrm{T}$ cell function as measured by the lymphoproliferative response (LPR) to $M$. tuberculosis (PPD, H37Ra-M. tuberculosis and lipoarabinomannan LAM)-antigens to determine the effect of HIV-infection in TB transmission. We evaluated, so far 5 HIV-positive HC (173 to $378 \mathrm{CD}^{+} / \mathrm{mm}^{3}$; 4 of 5 PPD skin test negative; all BCG vaccinated) and as a control group $20 \mathrm{HIV}$-negative $\mathrm{HC}$ (800 to $950 \mathrm{CD}^{+} / \mathrm{mm}^{3} ; 10$ PPD skin test negative; all BCG vaccinated) matched by age and time of exposure to the tuberculous patients. The HIV-positive HC group showed little response to $M$. tuberculosis antigens, even regarding their PPD skin test status (stimulus index < 3). 19 PPD negative HC were followed by conversion of their PPD skin test 
for 4 to 12 months and re-analyzed by LPR. Nine of them showed increased skin test reaction greater than $10 \mathrm{~mm}$, a criterion for conversion. The LPR in these $9 \mathrm{HC}$ increased 5-10 fold. Interestingly, 5 PPD non-convertors HC showed an increased LPR, compatible to the PPD convertor. Of these, one of them had pulmonary tuberculosis on further evaluation, the other 2 are under clinical evaluation. These results suggest that DTH reactivity criteria for initiation of chemoprophylaxis may require re- evaluation for individuals at the highest risk in order to prevent tuberculosis.

We conclude that HIV-positive patients with tuberculosis have a reduced immune response, and the failure of $\mathrm{CD}^{+} \mathrm{T}$ cells likely limits an effective immune response against $M$. tuberculosis, thus resulting in lower ability to control M. tuberculosis and survival.

Acknowledgements: to all the co-workers who are co-authors or participants of the cited studies. 